Referencia para citar este artículo: Díaz-Alzate, M. V. \& Mejía-Zapata, S. I. (2018). La mirada de los adolescentes al modelo de habilidades para la vida. Revista Latinoamericana de Ciencias Sociales, Niñez y Juventud, 16(2), 709-718. doi:https://doi.org/10.11600/1692715x.16205

\title{
La mirada de los adolescentes al modelo de habilidades para la vida*
}

\author{
MAGDa Victoria Díaz-ALZate ** \\ Profesora-investigadora Universidad Católica Luis Amigó, Colombia. \\ SANDRA ISABEL MEJÍA-ZAPATA ${ }^{* * *}$ \\ Profesora-investigadora Universidad Católica Luis Amigó, Colombia.
}

\begin{abstract}
Artículo recibido en mayo 2 de 2017; artículo aceptado en septiembre 18 de 2017 (Eds.)
\end{abstract}
La escuela puede promover activamente el pensamiento crítico, así como la habilidad y el coraje de expresarlo, aunque disienta de los demás.

- Nussbaum (2010, p. 74)

- Resumen (descriptivo): el enfoque de habilidades para la vida, es recomendado por la Organización Panamericana de la Salud (OPS) como modelo para la prevención de conductas de inicio temprano del consumo de sustancias psicoactivas. En este sentido, es importante identificar el estado del desarrollo de estas habilidades en los adolescentes, con el propósito de reflexionar sobre las experiencias de aprendizaje que promueven las instituciones educativas, vinculadas a las capacidades para una vida sana. Este artículo presenta una aproximación crítica a la noción de habilidades para la vida y dos categorías emergentes de investigación, como parte de los resultados: el enfoque de habilidades para la vida: percepciones críticas de los adolescentes, y el desarrollo de las habilidades sociales en los adolescentes: una mirada al contexto.

Palabras clave: habilidad, educación, desarrollo, configuración, adolescencia (Tesauro de Ciencias Sociales de la Unesco).

\section{What adolescents think of the Life Skills model}

- Abstract (descriptive): The Life Skills approach is recommended by Pan American Health Organization (Paho) as a model for preventing behaviors of early consumption of psychoactive substances. In this sense, it is important to identify the state of this abilities development in adolescents, with the purpose of reflecting about learning experiences, which promote educative institutions; those skills are tied in with abilities for a healthy life. This article presents a critical approach to the notion of life skills and two emergent research categories, as part of the results: Life Skills approach: critical

Este artículo de reflexión presenta dos categorías emergentes halladas en la investigación culminada: «Desarrollo de Habilidades para la vida en la prevención de las adicciones de adolescentes de 12 a 14 años escolarizados en Instituciones Educativas públicas de la ciudad de Medellín». La investigación de corte hermenéutico, fue realizada por las autoras y financiada por la Universidad Católica Luis Amigó, entre el 2 de febrero y el 20 de noviembre de 2016. Código del proyecto SIGP: 42409. Área de conocimiento: Sociales Interdisciplinarias; subárea: Temas Especiales (Estudios psicosociales sobre juventud y educación).

** Magister en Educación y Desarrollo Humano. Profesora-investigadora Universidad Católica Luis Amigó. Orcid: 0000-0002-7712-8462. Correo electrónico:magda.diazal@amigo.edu.co

**** Magister en Intervenciones Psicosociales, Profesora-Investigadora Universidad Católica Luis Amigó. Medellín-Colombia. Orcid: 0000-00026368-314X. Correo electrónico: sandra.mejiaza@amigo.edu.co 
perceptions from adolescents, and a contextual overview of the development of social skills in this population.

Key words: Skill, Education, Development, Configuration, Adolescence(Unesco Social Sciences Thesaurus).

\section{A visão dos adolescentes ao modelo de habilidades para a vida}

- Resumo (descritivo): o enfoque de habilidades para a vida, é recomendado pela Organização Pan-americana da Saúde (OPS) como um modelo para prevenir comportamentos de consumo precoce de substâncias psicoativas. Neste sentido, é importante identificar o estado do desenvolvimento dessas habilidades nos adolescentes, com o propósito de refletir sobre as experiências de aprendizagem que promovem as instituições educativas ligadas às capacidades para uma vida saudável. Este artigo apresenta uma abordagem crítica da noção de habilidades para a vida e duas emergentes categorias de pesquisa, como parte dos resultados: o enfoque de habilidades para a vida: percepções críticas dos adolescentes, e o desenvolvimento das habilidades sociais nos adolescentes: uma visão do contexto.

Palavras-chave: habilidade, educação, desenvolvimento, configuração, adolescentes (Thesaurus de Ciências Sociais da Unesco).

-1. Introducción. -2. Metodología. -3. Una aproximación crítica a la noción de habilidades para la vida. La comprensión de la configuración de subjetividades. -4. Enfoque de habilidades para la vida: percepciones críticas de los adolescentes. $\mathbf{- 5}$. Desarrollo de las habilidades sociales en los adolescentes: una mirada al contexto. -6. Consideraciones finales. -Lista de referencias.

\section{Introducción}

Este artículo se deriva de la investigación «Desarrollo de habilidades para la vida en la prevención de las adicciones de adolescentes de 12 a 14 años escolarizados en instituciones educativas públicas de la ciudad de medellín», realizada en el año 2016, y cuya pretensión era realizar una valoración del estado actual de las habilidades para la vida de jóvenes de la población nombrada, con el fin de generar líneas de prevención en el tema de las adicciones que contribuyan a prácticas más saludables, todo ello debido a que existe, para el Estado colombiano, la necesidad imperante de realizar programas de prevención que disminuyan el uso de sustancias o retarden la edad de inicio en el uso de las mismas, pues en el último Estudio Nacional de Consumo de Sustancias Psicoactivas en Colombia, realizado en todo el país (2013), y en el Reporte de Drogas de Colombia (Ministerio de Justicia y del Derecho, \& Observatorio de Drogas de Colombia, 2016), se muestra a la población juvenil como una de las principales poblaciones para la intervención.

No obstante, en el desarrollo de este trabajo se halló una fractura entre lo planteado desde los Lineamientos para una política pública frente al consumo de drogas, de la Comisión Asesora para la Política de Drogas en Colombia (Observatorio de drogas de Colombia, 2013) y las experiencias de los jóvenes cuando participan en el desarrollo de las actividades encaminadas hacía la prevención del consumo y el desarrollo de habilidades para la vida, según el enfoque que propone la Organización Panamericana de la Salud (OPS, 2001). Lo que se abordará a continuación serán los cuestionamientos a la noción de habilidades para la vida y a los modos de acercamiento y acompañamiento de trabajo con adolescentes en programas de prevención en la instituciones educativas. Estos aspectos se organizan en dos categorías emergentes complejas, que reorientaron los propósitos previos de la investigación, toda vez que permiten comprender aspectos más profundos que tienen que ver con discursos hegemónicos de las organizaciones internacionales como la OPS, propios de los modelos de mediciones del déficit (Gergen, 2007), de la homogenización de la conducta y de las prácticas intervencionistas, ligadas a la higienización. 
En el análisis de los relatos de los adolescentes participantes, se encuentran percepciones críticas del enfoque de habilidades para la vida y la necesidad de comprender los contextos locales en los que viven estos adolescentes, en quienes se pretende la aplicación del enfoque propuesto por la OPS. Estos relatos sirvieron de pretexto para la reflexión crítica frente a este modelo, que se viene implementando en las instituciones educativas (las que se seleccionaron para la investigación).

Lo descrito tiene implicaciones en la reconfiguración de propuestas educativas, lo cual demanda la incorporación de estrategias que se consoliden como aportantes y valiosas para la formación integral de niños, niñas y adolescentes, pero con una comprensión de configuración de subjetividades pluralista. Aquí Martha Nussbaum ofrece una alternativa importante a partir del enfoque de las capacidades, puesto que contiene en sí misma, la mirada pluralista en la pregunta del mismo enfoque: «¿qué es capaz de hacer y de ser cada persona?»(Nussbaum, 2012, pp. 38-40), que si bien no se desarrolla en este artículo como alternativa para la construcción de otro enfoque, podría servir de entrada a futuras propuestas.

El modelo propuesto por la OPS en 2001 define las habilidades como «destrezas para permitir que los adolescentes adquieran las aptitudes necesarias para el desarrollo humano y para enfrentar en forma efectiva los retos de la vida diaria» (p. 5), lo cual implica dos asuntos críticos para que pueda ser adaptado a cualquier población. La primera tiene que ver con equiparar habilidad con destreza, y resultante de un proceso de aprendizaje. Ello implica que la adquisición de habilidades para la vida, requiere de otro a quien se le supone un saber-hacer y un saber-vivir, quien haría las veces de facilitador para que otros adquieran estas destrezas. Aquí, es importante considerar la fundamentación teórica de la OPS para el enfoque que proponen, toda vez que se centra en concepciones del desarrollo humano evolucionistas, que se enmarcan en lo que Gergen (2007), nombra como discursos del déficit. De allí la imperiosa necesidad de construir propuestas intervencionistas para la normalización de la conducta.

El otro asunto crítico, es considerar que pre-existen unas aptitudes necesarias para el desarrollo humano, cuando esta organización propone que las habilidades para la vida se agrupan en tres grandes categorías y cada una de ellas se desglosa en habilidades específicas (OPS, 2001): habilidades sociales: comunicación asertiva, empatía y relaciones interpersonales; habilidades cognitivas o de pensamiento: toma de decisiones, solución de problemas, pensamiento creativo y pensamiento crítico; y Habilidades del control de emociones: conocimiento de sí mismo, manejo de sentimientos y manejo de tensiones y estrés. Esta propuesta pone de nuevo en evidencia una concepción evolucionista del desarrollo humano, centrado en la necesidad de homogenización de conductas, a través de contenidos discursivos que propenden por mostrar una sola manera de alcanzar capacidades para tener un desarrollo adecuado.

Este enfoque concibe un desarrollo humano idóneo de ser medido en términos de la eficacia para la vida en común y para las instituciones, si se tiene presente que, como lo refieren Mangrulkar, Whitman y Posner (2001), desde el surgimiento del modelo de habilidades para la vida, su implementación ha estado encaminada al «desarrollo de jóvenes, formación de valores, prevención de embarazos, planificación de la vida, aprendizaje social y emocional, educación para la salud o iniciativas para la prevención del abuso de las drogas» (p. 7), lo cual es susceptible de ser medido en términos de indicadores.

Si bien este enfoque ha resultado pertinente en algunos casos en Colombia como la experiencia referida de Fe y Alegría (Mantilla-Castellanos, 2001), cabe anotar que aún bajo la eficacia que se le confiere no debe ser considerado como un modelo finalizado, puesto que las condiciones sociales, políticas, económicas y contextuales se transforman y exigen repensar lo estructurado para los procesos educativos de nuevas maneras de configuración subjetiva de niños, niñas y adolescentes. Asimismo, es importante contemplar otros enfoques sobre el desarrollo humano, menos en términos de crecimiento o acumulación de destrezas, y más desde las singularidades de los sujetos y de los contextos. 


\section{Metodología}

La investigación se enmarca en el estudio cualitativo, con un enfoque epistemológico hermenéutico, que privilegia la comprensión de las experiencias vividas por los sujetos participantes a partir del ejercicio interpretativo. Al decir de Herrera (2009), la experiencia hermenéutica se refiere al lugar de la interpretación que tiene siempre el ser humano (p. 121) y para Gadamer (1998), «La capacidad de comprensión es (es una) facultad fundamental de la persona que caracteriza su convivencia con los demás y actúa especialmente por la vía del lenguaje y del diálogo» (p. 319).

Para alcanzar el objetivo de la investigación, que fue identificar las habilidades para la vida de adolescentes escolarizados en edades de 12 a 14 años de cinco instituciones educativas públicas de la ciudad de Medellín, con el propósito de establecer líneas preventivas en prácticas de riesgo, se seleccionaron tres instituciones educativas públicas de la ciudad de Medellín, que cumplieran el criterio de accesibilidad, con los estudiantes de psicología de la Universidad Católica Luis Amigó (Funlam) que se encontraban en práctica profesional allí, y se contó con la participación de 75 estudiantes - 25 por cada institución-, con las edades definidas en el objetivo.

Para la generación de la información, se realizaron dos grupos focales en cada una de las tres instituciones, después de la aplicación de un cuestionario adaptado, al cual se realiza un pilotaje por parte de docentes investigadores del grupo de investigación Farmacodependencia y otras Adicciones de la Funlam, y que definía 60 afirmaciones: 18 sobre habilidades sociales, 21 sobre habilidades cognitivas y 21 sobre habilidades emocionales, con una escala de 1 a 4: 1 totalmente en desacuerdo, 2: medianamente de acuerdo, 3: de acuerdo y 4: totalmente de acuerdo. Este último permitió una valoración de las habilidades para la vida en términos cuantitativos, como un primer acercamiento para definir las preguntas guía de los grupos focales, que, en última instancia, generan la información más relevante para el análisis de la información, de acuerdo al enfoque cualitativo propuesto.

El proceso de triangulación se realiza con la información que arroja la encuesta, los relatos de los adolescentes que se generan en los grupos focales, y el rastreo de literatura científica y antecedentes investigativos, que se sistematizan en el programa de Atlas.ti para su categorización y análisis.

\section{Consideraciones éticas}

Esta investigación contempló las directrices estipuladas por el Ministerio de Salud Nacional de Colombia (Resolución $N^{\circ}$ 8430, Ministerio de Salud, 1993), sobre la investigación con participantes humanos y el nivel de riesgo que implica; para ello, las investigadoras, a través del consentimiento informado, dan a conocer los alcances del proyecto, los derechos de los participantes y los riesgos mínimos que se corrían — se considera que la investigación es de riesgo bajo-. Asimismo, por tratarse de menores de edad, el consentimiento es firmado por los padres o en su defecto quien hacía las veces de acudiente del adolescente en la institución educativa, quien es el representante legal.

En este compromiso ético, las investigadoras advierten que la información generada sólo será utilizada con fines académicos y que la identidad, tanto de las instituciones educativas como de los participantes, se mantendría reservada.

\section{Una aproximación crítica a la noción de habilidades para la vida. La comprensión de la configuración de subjetividades}

Comprender la configuración de subjetividades como proceso dinámico, que implica la construcción de sentidos frente a las acciones humanas (González-Rey, 2007), supone la concepción de sujeto como activo, capaz de generar procesos de subjetivación y reflexión (González-Rey, 2007), en contraposición al sujeto solamente conductual, que responde frente a estímulos, discursos o acciones para adaptarse a las consideraciones del orden social establecido en un momento histórico preciso. La configuración de subjetividades propone que la capacidad de respuesta no se debe a 
una estimulación o a un proceso de transmisión de información, sino que el sujeto se supone capaz de interpretar los discursos para reflexionar sobre los mismos y construir sentidos subjetivos. Esta comprensión de las capacidades humanas para la configuración de subjetividades, pone en tensión el propósito del enfoque de habilidades para la vida, toda vez que reconoce la individuación y la capacidad de construcción de sentidos de las acciones por parte de los sujetos.

La configuración de subjetividades no se desliga de la cultura, aunque se conciba como un proceso individual, contiene en sí misma los sentidos que se han construido en la cultura de pertenencia del sujeto, en un proceso de interacción. La importancia de comprender la subjetividad como una configuración, está en la posibilidad de los sujetos de interpretar los sentidos que se han construido en la cultura, comprenderlos y reconfigurarlos de manera subjetiva.

La comprensión de los sujetos como capaces de configurarse, a partir de unas relaciones intersubjetivas, se opone al concepto de desarrollo humano como proceso de crecimiento, acumulativo y susceptible de medición estandarizada y generalizable, que estaría en el fundamento teórico del modelo propuesto por la OPS para el desarrollo de habilidades para la vida, que habla de etapas del desarrollo en la infancia y la adolescencia (2001).

Sibien es importante el conocimiento de hitos del desarrollo evolutivo de niños, niñas y adolescentes desde las teorías de los ciclos vitales, no puede constituirse como manera única de comprensión de sus vivencias y sus aprendizajes. De entrada, los conceptos de desarrollo y habilidades equiparadas a destrezas que se adquieren, enmarca el modelo de habilidades para la vida en el paradigma positivista de comprensión de las realidades y de los sujetos, y evidencia una mirada reduccionista del desarrollo humano, de allí que pueda decirse que el enfoque propuesto como modelo por la OPS, contenga un discurso propio del desarrollo de competencias, es decir, del orden de lo que está concebido como discurso competente. Al decir de Chauí (2017):

el discurso competente como discurso de conocimiento entra en escena para intentar devolver a los objetos socioeconómicos y socio-políticos la cualidad de sujetos que les fuera sustraída. Esa tentativa se realiza a través de la competencia privatizada. Invalidados como seres sociales y políticos, los hombres serían revalidados a través de una competencia que les corresponde en tanto sujetos individuales o personas privadas. (pp. 261-262)

Esta concepción de desarrollo humano en términos de competitividad, propio del sistema económico capitalista de medición productiva y eficaz, releva la condición humana de la diferencia y la posibilidad de pluralismo, lo cual puede considerarse excluyente y perpetuador de discursos hegemónicos de poder, que desconocen las capacidades humanas como posibilidades, para ubicarlas en el umbral de la competencia entre los sujetos, sobre estándares de calidad.

\section{Enfoque de habilidades para la vida: percepciones críticas de los adolescentes}

El modelo de la OPS (2001), ha tomado fuerza en la educación para implementar estrategias que favorezcan el desarrollo de las habilidades para la vida, como factores protectores frente a conductas de riesgo como el consumo de sustancias psicoactivas en adolescentes. Sin embargo, uno de los hallazgos relevantes de esta investigación, que permitió la caracterización del estado de desarrollo de estas habilidades en los estudiantes participantes, permite dilucidar que existe una brecha entre las intenciones del modelo, las estrategias implementadas y el resultado.

Los relatos de los adolescentes, generados en los grupos focales, permiten hacer lectura analítica e interpretativa de las percepciones que tienen ellos sobre los diferentes programas implementados en las Instituciones Educativas con los fines descritos; es así como uno de los participantes, menciona: «las charlas para que no metamos yerba [sic] son muy casposas, uno no aprende de la vida con palabrería», cuando en el grupo focal se discute sobre el programa de prevención de la institución.

Esta capacidad para construir significados sobre las estrategias que vienen implementando las Instituciones Educativas en el marco de la aplicación del modelo del desarrollo de habilidades para la vida de la OPS, precisa expandir la comprensión de los jóvenes, en tanto se revelan como sujetos 
activos en los diferentes procesos educativos. Su capacidad para evaluar las prácticas educativas, permite visibilizarlos en su proceso configurativo, no como sujetos conductuales que responden a estímulos, sino como capaces de disentir frente a las imposiciones de los sistemas. Esta lectura de los discursos juveniles, favorece en gran medida poner en crisis lo establecido como modelos de desarrollo de habilidades para la vida, como es el enfoque propuesto por la OPS y acomodado a todos los contextos latinoamericanos, desconociendo, que incluso en la región, hay diferencias entre países, entre ciudades y entre sujetos.

Asimismo, otro de los adolescentes frente a la discusión sobre el tema de la empatía, narra: «No veo que en el colegio uno pueda aprender a ponerse en el lugar de los compañeros, sabiendo que todo lo que nos ponen a hacer es para que competamos [sic]; y tampoco uno aprende esas cosas como aprende a sumar. » (Participante)

Si bien son dos experiencias de instituciones diferentes y con temas de discusión distintos, permiten un acercamiento para comprender las percepciones de los adolescentes. Específicamente, este último relato hace explícito el sentir del participante frente a estrategias de enseñanza de habilidades para la vida, y el anterior frente a programas de prevención del consumo, y vislumbran de manera implícita, la crítica frente a la manera en la que se ha venido desarrollando, tanto la estrategia para el desarrollo de habilidades, como los programas de prevención. Si bien la investigación no se centró en los programas de prevención de consumo, vale la pena resaltar que la dinámica misma del ejercicio, propició que emergiera el tema y amplió la posibilidad de análisis de las investigadoras.

Así, existe una recurrencia en la manera en la que los adolescentes se refieren al activismo en las dinámicas institucionales para impartir estrategias que favorezcan la prevención del consumo de sustancias psicoactivas, que no conversan con las expectativas y las experiencias de vida de estos adolescentes frente a este asunto, puesto que ven en estos programas preventivos, irrelevancia cuando en lo que se concentran es en identificar como factores de riesgo, la relación con sus pares:

«es verdad, la psico habla de que dejemos a los amigos que consumen y que nos hagamos parceros [sic] de los que, para ella, son juiciosos, pero no sabe que aquí hay más de uno todo solapado [sic] y no vamos a dejar a los parceros de siempre porque les guste meter.» (Participante)

La escuela como escenario propicio para la socialización de niños, niñas y adolescentes, no escapa al interés de disciplinamiento de los cuerpos y de las conductas, lo cual está contemplado en el afán higienista de las organizaciones que se encargan del desarrollo de temas sobre salud mental. Esta postura que toma fuerza en el siglo XIX (Viñao, 2010; Cordoví-Núñez, 2012), con disciplinas como la medicina y la psicología, se introduce en el proceso escolar y aún en la actualidad se puede leer en diferentes programas intervencionistas de instituciones estatales, organizaciones no gubernamentales y otros estamentos, que si bien tratan de expresarse en una terminología que podría leerse como constructivista, desde las posturas teóricas y los intereses por la salud mental con la división entre normalidad y anormalidad, continúan perpetuando los discursos de inclusión y exclusión según criterios estandarizados de medición del desarrollo.

La Unicef (2002, como se citó en Fundación Jacobs, 2011), define las habilidades como «recursos psicosociales e interpersonales que ayudan a la gente a tomar decisiones con conocimiento de causa, comunicarse de forma efectiva y desarrollar unos recursos para manejar y autogestionar una vida saludable y productiva» (p. 10). Aquí es posible leer una de las características del sistema neoliberal (Castro, 2009), si se advierte que, en este sistema,

Hay un imperativo de la libertad que fuerza a los individuos a hacerse cargo de sí mismos. Es en este contexto que se promueve y se impone en todo orden de materias la lógica de lo privado, lo personal o lo individual, contra las dimensiones de lo público, lo común o lo intersubjetivo. Un ejemplo muy claro de esta racionalidad y que afecta directamente al campo de la medicina y la salud es el modo de concebir la política social. (Castro, 2009, p. 21) 
En este sentido, se precisa una mirada crítica al enfoque tradicionalmente propuesto para el desarrollo de habilidades para la vida, de tal manera que se incluya en sus consideraciones, la comprensión de formación continua y permanente, más allá de los procesos de enseñanza de conceptos y actitudes, por medio de talleres específicos y por tiempos de aplicación, como lo sugieren diferentes lineamientos como Una propuesta educativa para la promoción del desarrollo humano y la prevención de problemas psicosociales (Mantilla, 2001); Lineamientos para la promoción de la salud con-sentido humano de Antioquia (Gobernación de Antioquia, 2010); Módulo habilidades para la vida (Gobernación de Antioquia, 2014); entre otras.

\section{Desarrollo de las habilidades sociales en los adolescentes: una mirada al contexto}

Según los hallazgos arrojados por los cuestionarios, las habilidades sociales, es el grupo que en menor escala tiene desarrollado los adolescentes del estudio, lo cual toma fuerza cuando se analizan los diferentes relatos. El cuestionario arrojó que el $60 \%$ de los adolescentes seleccionó la opción 2 de la escala, es decir, medianamente de acuerdo; mientras que el 20\% se dirigió a la opción 3, que indica que están de acuerdo y los participantes restantes, eligieron la opción 1, que define totalmente en desacuerdo. Si bien esta valoración no es central para los resultados de la investigación, permitió ahondar en los grupos focales sobre temas como las relaciones interpersonales, la capacidad de escucha y de diálogo, y la posibilidad de reconocimiento de los otros.

La importancia de este análisis, radica en que en la adolescencia, los procesos de socialización y de relacionamiento con pares, influyen directamente en los comportamientos, puesto que, es un período en el cual, hay un desplazamiento del grupo familiar, por este grupo de identificación.

En este sentido, uno de los participantes frente a la pregunta por las relaciones con los compañeros y la búsqueda de apoyo en momentos de tensión, relata: «no me gusta de amucho [sic] pedir favores ni hablar de mis problemas con los del salón; no creo que me entiendan cuando me dan ganas de pegarme un vuelo (refiriéndose a los momentos en los que consume marihuana)».

Mangrulkar et al. (2001), advierten que «la iniciativa global para la salud escolar y la red de trabajo en las escuelas para promover la salud han adoptado las habilidades para la vida como una estrategia prioritaria para la salud escolar» (p. 7), pero es indudable que se requiere la comprensión de contextos en los que existen factores de riesgo mayores, para ahondar en iniciativas diferenciadoras que tengan presentes las particularidades contextuales y sociales, como se evidencia en otro relato:

«Vea, eso de que uno puede apoyarse en la family [sic] cuando tiene problemas en el colegio, o en los compañeros cuando la casa está prendida a golpes, es difícil. Uno encuentra más brothers [sic] en la calle que con un pase lo escuchan a uno.» (Participante)

La adolescencia es un momento del ciclo vital crucial para la configuración de la subjetividad y la identidad, y la relación con los pares constituye el proceso de socialización más importante, en tanto permite la identificación con intereses comunes y la posibilidad de establecer redes de apoyo, sean éstas concebidas como factores protectores o de riesgo. Al respecto, Krauskopf (1998), dice que El desarrollo juvenil se da en una delicada interacción con los entes sociales del entorno; tiene como referente no sólo la biografía individual, sino también la historia y el presente de su sociedad. Es el período en el que se produce con mayor intensidad la interacción entre las tendencias individuales, las adquisiciones psicosociales, las metas socialmente disponibles, las fortalezas y desventajas del entorno. (p. 119)

Precisamente estas características del momento vital de la adolescencia, son las que resaltan la importancia de la consolidación de relaciones interpersonales, que a su vez sean recursos preventivos de conductas de riesgo como el consumo de sustancias psicoactivas, puesto que, como lo nombra D’Aloisio (2017), «los jóvenes construyen diversas prácticas y relaciones de sociabilidad en los espacios que habitan, como la escuela, el barrio, la calle, las redes sociales de comunicación y otros lugares de encuentro o esparcimiento» (p. 105). En este sentido, el relato anterior llama la atención 
de manera particular, toda vez que pone de manifiesto la relación con otros, pero no necesariamente como agentes protectores, más bien, como posibles generadores de riesgos para las adicciones.

Aquí se hace prudente comprender la concepción de Soler-Roca (2014), respecto al trabajo educativo:

Todo trabajo educativo es un trabajo situado. Debe atender las necesidades del desarrollo de seres concretos, miembros de un grupo humano determinado, en un lugar geográfico y ecológico sin igual, en un momento histórico preciso, en un ámbito político, social, cultural, lingüístico con características que le son propias. Lo resumo diciendo que la educación está caracterizada por su historicidad. Así ha sido siempre; así seguirá siendo. (p. 45)

Es imperativo entonces, comprender la importancia de un proceso formativo transversal en la educación formal, respecto a las habilidades sociales, no sólo desde la enseñanza de las mismas, sino más bien, a partir de la propensión por su aprehensión, puesto que es evidente, en el caso de los adolescentes participantes de esta investigación, que, si bien construyen relaciones sociales, no hacen juicio valorativo de lo que puede constituir un riesgo. Si bien en la adolescencia la transgresión puede aparecer como característica, también es innegable que es posible que desarrollen juicios valorativos sobre lo que es el bien y el mal, en términos del desarrollo moral.

Ahora bien, si se entienden estas habilidades como destrezas, no es posible contemplarlas más allá del adoctrinamiento para la perpetuación de la vida cívica en términos educacionales, es decir, desde el moldeamiento de las corporalidades para el mantenimiento del orden social establecido. En este sentido, se mantiene el modelo tradicional de la educación, el cual concibe a los sujetos desde la perspectiva conductual, lo cual los reduce a la expresión de educables, pero desconoce la capacidad de actuación, que sería el reconocimiento de su subjetividad. Para expandir las posibilidades de actuación en la formación de los niños y adolescentes en términos de la vida social, habrá que deconstruir también la concepción adoctrinante de las habilidades sociales.

La escuela debe ser entonces aquel lugar en el que se construyan preguntas sobre la vida, más que la confluencia de represores y adoctrinadores de esta vida, puesto que es privilegiada para la socialización entre diferentes, para el reconocimiento de la diversidad y por tanto, puede contener las condiciones para la construcción colectiva de sentidos de vida, de mundo compartido y de concepto de pluralidad. Tal vez así, más que estrategias para enseñar lo que no puede ser enseñado como vivir, se pueda configurar como espacio para preguntarse por lo que es vivir en lo social, y no como el lugar que busca la certeza en el desarrollo de destrezas sociales.

\section{Consideraciones finales}

El modelo para el desarrollo de habilidades para la vida propuesto por la OPS (2001), ha venido posicionándose en las instituciones educativas como parte de las estrategias para la promoción de estilos de vida saludables y la prevención de conductas de riesgo como el consumo de sustancias psicoactivas, entre otros. Si bien el modelo no es susceptible de ser descartado por los hallazgos de la investigación que dio sentido a este artículo, es importante reconocer que, escuchar las voces de los adolescentes que hacen parte de las instituciones educativas públicas, en las cuales se tiene el modelo para el desarrollo de habilidades para la vida, compromete la manera en que se está comprendiendo la configuración subjetiva de los jóvenes en la actualidad y la capacidad para leer los fenómenos sociales como factores coadyuvantes en esta configuración.

La reflexión sobre la época, el contexto y la configuración de nuevas subjetividades, permitirá que modelos de intervención, como el del desarrollo de habilidades para la vida, sea sometido a validación, de acuerdo a las condiciones de la población, y no con el fin de replicar maneras de estar en el mundo de los jóvenes, cuando las estas condiciones no son estandarizadas y equitativas en el mundo.

Según los hallazgos, las habilidades sociales, es el grupo que en menor escala tiene desarrollado los adolescentes del estudio; por tanto, es relevante comprender la importancia de un proceso formativo 
transversal en la educación formal, respecto a las habilidades sociales, no sólo desde la enseñanza de las mismas, sino a partir de la propensión por su aprehensión.

Si se tiene en cuenta que los hallazgos de esta investigación sugieren que el enfoque centrado en la enseñanza de las habilidades para la vida no es suficiente, para el abordaje del fenómeno del consumo de sustancias psicoactivas y de la prevención de otras conductas de riesgo en adolescentes, se precisa una mirada crítica al enfoque tradicionalmente propuesto, de tal manera que se incluya en sus consideraciones, la comprensión de formación continua y permanente y la reformulación del modelo con una perspectiva de formación integral de niños, niñas y adolescentes, transversal en los currículos de las instituciones educativas.

El modelo tradicional de la educación, ha impregnado todo aquello que se implementa en la Instituciones Educativas, haciendo que se equipare educación con adoctrinamiento. Sin embargo, esta investigación pone de relieve la incomunicabilidad que existe entre las pretensiones educacionales del modelo y la necesidad cada vez más visible y plausible de los sujetos a ser reconocidos en su diferencia y como un fin en sí mismos. En este caso, vale la pena conciliar una alternativa para la construcción de alternativas formativas en la educación formal que favorezcan, más que el desarrollo de lo que se ha nombrado como habilidades para la vida, una construcción de vida en común que tenga por principio, la relación ética con los otros y la comprensión del «nosotros» como apuesta para una vida digna.

En este caso, el enfoque de las capacidades de Martha Nussbaum (2012), la comprensión de la relación ética en la educación que se opone al adoctrinamiento propuesto por Duch y Mèlich (2009), tener en cuenta la mirada de González-Rey (2007) sobre las subjetividades y la concepción de desarrollo humano más allá de la impronta productivista, podrían consolidarse como componentes epistemológicos y críticos para la construcción de estrategias educativas, que más que interesarse por el desarrollo de unas habilidades propuestas para el adoctrinamiento de las corporalidades, favorezca la vida digna, la construcción colectiva, el reconocimiento entre los seres humanos como fines en sí mismos, la compasión y el cuidado de sí, de los otros y de lo otro no humano.

\section{Lista de referencias}

Castro, R. (2009). Capitalismo y medicina. Los usos políticos de la salud. Ciencia Política 7, 7-25. Chauí, M. (2017). El discurso competente. En N. Prigorian, \& C. Díaz-Orozco(Eds.), Representaciones, emergencias y resistencias de la crítica cultural. Mujeres intelectuales en América Latina y el Caribe, (pp. 253-263). Buenos Aires: Clacso. Recuperado de:

http://biblioteca.clacso.edu.ar/clacso/se/20170728014425/Representaciones.pdf

Cordoví-Núñez, Y. (2012). Cuerpo, pedagogía y disciplina escolar en Cuba: dispositivos de control desde los discursos higienistas (1899-1958). Tzintzun. Revista de Estudios Históricos, 56, 93136. Recuperado de:

http://www.scielo.org.mx/pdf/tzintzun/n56/n56a3.pdf

D’Aloisio, F. (2017). Jóvenes y sociabilidad escolar: aprendizajes que sostienen determinado orden social. Revista Latinoamericana de Ciencias Sociales, Niñez y Juventud, 15(1), 101-115. doi: 10.11600/1692715x.1510509112016

Duch, L., \& Mèlich, J. C. (2009). Ambigüedades del amor. Antropología de la vida cotidiana. Madrid: Trotta.

Fundación Jacobs. (2011). Seguimiento y evaluación de las habilidades para la vida orientadas al desarrollo de la juventud. Berna: Layout Prepress Printing.

Gadamer, H. G. (1998). Verdad y método II. Salamanca: Sígueme.

Gergen, K. (2007). Las consecuencias culturales del discurso del déficit. En A. Estrada-Mesa, \& S. Díazgranados-Ferráns (Comps.), Construccionismo social aportes para el debate y la práctica, (pp. 281-310). Bogotá, D. C.: Uniandes. 
Gobernación de Antioquia. (2010). Lineamientos para la promoción de la salud con-sentido humano en el departamento de Antioquia. Medellín: Dirección Seccional de Salud y Protección Social de Antioquia. Recuperado de:

https://www.dssa.gov.co/index.php/descargas/733-libro-lineamientos-promocion-salud/file

Gobernación de Antioquia. (2014). Módulo de habilidades para la vida. Guía para el trabajo con adolescentes y jóvenes. Medellín: Secretaría Seccional de Salud y Protección Social de Antioquia. Recuperado de:

https://www.dssa.gov.co/index.php/descargas/877-modulo-habilidades-para-la-vida/file

González-Rey, F. (2007). Posmodernidad y subjetividad: distorsiones y mitos. Revista de Ciencias Humanas 37, 7-26. Recuperado de:

http://revistas.utp.edu.co/index.php/chumanas/article/view/1135/623

Herrera, J. D. (2009). La comprensión de lo social. Horizonte hermenéutico de las ciencias sociales. Bogotá, D. C.: Ánthropos.

Krauskopf, D. (1998). Dimensiones críticas en la participación social de las juventudes. Participación y Desarrollo Social en la Adolescencia. San José: Fondo de Población de Naciones Unidas. Recuperado de:

http://bibliotecavirtual.clacso.org.ar/ar/libros/cyg/juventud/krauskopf.pdf

Mangrulkar, L., Whitman, C. V., \& Posner, M. (2001). Enfoque de habilidades para la vida para un desarrollo saludable de niños y adolescentes. Washington, D. C.: Organización Panamericana de la Salud. Recuperado de:

http://148.204.52.13/i/bibliotecaDase/2000/Acervo/AcervoVirtualPsicosocial/ PsicosocialAcervoLecturas/Habilidades.pdf

Mantilla-Castellanos, L. (2001). Habilidades para la Vida. Una propuesta educativa para la promoción del desarrollo humano y la prevención de problemas psicosociales. Bogotá, D. C.: Fe y Alegría. Recuperado de:

http://www.feyalegria.org/images/acrobat/72979810510810510097100101115_849.pdf

Ministerio de Justicia y del Derecho, \& Observatorio de Drogas de Colombia. (2016). Reporte de Drogas de Colombia. Bogotá, D. C.: Legis.

Ministerio de Salud. (1993). Resolución número 8430. Bogotá, D. C.: Ministerio de Salud. Recuperado de:

https://www.minsalud.gov.co/sites/rid/Lists/BibliotecaDigital/RIDE/DE/DIJ/RESOLUCION8430-DE-1993.PDF

Nussbaum, M. (2010). Sin fines de lucro. Por qué la democracia necesita de las humanidades. Buenos Aires: Katz.

Nussbaum, M. (2012). Crear capacidades. Madrid: Paidós.

Observatorio de drogas de Colombia. (2013). Lineamientos para una política pública frente al consumo de drogas. Recuperado de:

http://www.odc.gov.co/Portals/1/publicaciones/pdf/destacados/comision_asesora_politica_ drogas_colombia.pdf

Organización Panamericana de la Salud-OPS. (2001). Enfoque de habilidades para la vida para un desarrollo saludable de niños y adolescentes. Washington, D. C.: Recuperado de: http://148.204.52.13/i/bibliotecaDase/2000/Acervo/AcervoVirtualPsicosocial/ PsicosocialAcervoLecturas/Habilidades.pdf

Soler-Roca, M. (2014). Educación, resistencia y esperanza. Buenos Aires: Clacso. Recuperado de: http://biblioteca.clacso.edu.ar/clacso/se/20140718012935/SolerRoca.pdf

Viñao, A. (2010). Higiene, salud y educación en su perspectiva histórica. Educar, 36, 181-213. Recuperado de: http://www.scielo.br/pdf/er/n36/a13n36.pdf 